\title{
Le jeu de rôle dans l'enseignement supérieur pour développer l'autonomie et la motivation des étudiants
}

\author{
LAURENT BRISSON \\ IMT Atlantique, Lab-STICC, UMR CNRS 6285, Brest, France \\ laurent.brisson@imt-atlantique.fr \\ MARINE KARMANN \\ IMT Atlantique, F-29238 Brest, France \\ marine.karmann@imt-atlantique.fr
}

\section{Résumé}

L'informatique est une discipline exigeante à bien des niveaux, et son enseignement a suscité beaucoup de questionnements dans le domaine de la pédagogie. Quand s'ajoutent aux difficultés d'ordre disciplinaire, des éléments de complexité plus fort encore comme l'hétérogénéité d'un groupe d'étudiants (niveaux de compétences différents, formation et parcours professionnels différents, origines ethniques et culturelles différentes), les attendus des enseignants et des étudiants sont forcément mis à mal. Cet article propose l'évaluation d'un dispositif pédagogique par le jeu de rôle ayant pour but de prendre en compte les difficultés liées à l'altérité des étudiants et de permettre de les surmonter de manière ludique et motivante, en prenant en compte les spécificités de chacun au sein du groupe.

\section{Summary}

Computer science is a challenging discipline at many levels, and its teaching has raised many questions in the field of pedagogy. When complexity due to heterogeneity in a group of students (different levels of skills, curricula and professional experience) is added to disciplinary difficulties, expectations of both students and teachers are necessarily jeopardized. This article proposes an evaluation of a pedagogical experiment through Role-Playing Game whose purpose was to take into account the difficulties coming with students heterogeneity, and to 
overcome them in a playful and motivating way, considering the specificities of each and everyone within the group.

\section{Mots-clés}

Jeu de rôle, autonomie, motivation, travail en groupe, hétérogénéité.

\section{Key words}

Role play, autonomy, motivation, group work, heterogeneity.

\section{Introduction}

«Rien autant que le jeu n'exige d'attention, d'intelligence, (et) de résistance nerveuse. » (Roger Caillois, 1967)

La question de la diversité en enseignement n'est pas neuve et a fait l'objet de nombreux questionnements épistémologiques en pédagogie. Dans cet article, nous présentons un dispositif mis en place depuis 2015 à IMT Atlantique. Face à de nombreuses difficultés liées aux niveaux hétérogènes des étudiants en matière d'informatique, ou encore à la diversité de leurs expériences curriculaires, professionnelles et culturelles, nous avons mis en place un dispositif de jeu de rôle. Ce dispositif est joué en introduction à un enseignement de «Business Intelligence » accueillant de 40 à 60 étudiants selon les années et ayant lieu en $3^{\mathrm{e}}$ année de formation d'ingénieur (niveau Master 2) dans une filière de spécialisation en «Data Science ». Cet enseignement prend place après deux ans d'études au sein de l'école. Les formations de $3^{\mathrm{e}}$ année accueillent également des publics variés : étudiants admis sur titre d'école de commerce ou d'autres écoles d'ingénieurs. Ainsi, la connaissance du monde de l'entreprise est très variable, moins d'un an pour les étudiants ayant suivi un cursus traditionnel jusqu'à plusieurs années pour des étudiants déjà en possession d'un master, mais venant chercher une spécialisation. Le dispositif que nous proposons a pour objectif de prendre en compte l'hétérogénéité importante du groupe d'étudiants concerné ainsi que de répondre à la question suivante : comment concevoir un dispositif qui permettrait à la fois de prendre en compte l'altérité des niveaux et des parcours des étudiants, tout en donnant une vision d'ensemble des enjeux liés à un enseignement de spécialité en informatique?

Aux difficultés sociales et culturelles s'ajoutent souvent des difficultés disciplinaires: l'informatique étant un enseignement exigeant en terme aussi bien de savoir-faire que de savoir- 
être, il semble agir comme un révélateur de l'altérité des étudiants. Depuis les années 1980, les chercheurs notent la difficulté de l'enseignement et de l'apprentissage des fondamentaux de la programmation, le qualifiant de «tâche difficile, en particulier pour les novices » (Guéraud et Peyrin, 1988 ; Muratet et al., 2011).

Différentes hypothèses pour répondre à ce questionnement ont donc été formulées et mesurées par le biais d'une observation du déroulement des séances et d'un questionnaire distribué quelques semaines après l'expérience (voir Annexe 3). Les hypothèses de l'enquête étaient les suivantes : le recours au jeu de rôle permettrait de motiver les étudiants et de donner une vision d'ensemble du contenu de l'enseignement aux étudiants ; le recours au jeu de rôle aurait un impact bénéfique sur la constitution des groupes de travail en facilitant la gestion de l’hétérogénéité des étudiants.

Dans la section qui suit, nous détaillons le contexte et les problématiques identifiées, puis nous présentons en section 3 le dispositif qui traduit le jeu de rôle en activité pédagogique. En section 4 , nous évaluons notre première hypothèse sur la capacité à motiver et donner une vision d'ensemble du contenu, pour ensuite évaluer notre deuxième hypothèse sur l'impact de l'activité sur la constitution des groupes en section 5. Finalement, nous faisons un bilan de cette expérience en section 6 .

\section{Contexte et problématiques identifiées}

Ce retour d'expérience concerne un enseignement intitulé «Business Intelligence » qui est organisé sous forme d'apprentissage par projet (Brisson, 2015) et où les étudiants sont répartis en plusieurs groupes de cinq à six personnes. Cet enseignement vise à former de futurs ingénieurs qui seront amenés à concevoir ou réaliser des systèmes permettant la génération de tableaux de bord de suivi de la performance d'une organisation.

Les difficultés majeures dans l'apprentissage de ce domaine sont similaires à celles présentées par Muratet et al. (2011) concernant l'apprentissage de la programmation. Ces difficultés sont de deux ordres. Premièrement, les étudiants sont rapidement confrontés à des obstacles épistémologiques (Ginat, 2004) dont ils ne peuvent pas encore saisir toutes les implications et qui demandent une certaine capacité d'abstraction difficile à acquérir en début de formation (Seppälä et al., 2006). Deuxièmement, malgré une utilisation quotidienne des environnements informatiques par les étudiants dans des contextes sociaux ou de loisir, ils ne voient pas directement le lien qui s'opère entre leurs usages quotidiens et les dispositifs déployés dans les 
enseignements (Muratet et al., 2011). Dans le cadre de l'enseignement évoqué dans ce retour d'expérience, nous nous retrouvons donc confrontés au paradoxe important de l'enseignement de l'informatique établi par Muratet et al. (2011), qui consiste à devoir faire maîtriser aux étudiants des concepts et des connaissances que l'on ne peut vraiment maîtriser que lorsque l'on sait programmer.

Par ailleurs, on peut noter que les étudiants suivant cet enseignement ont des profils très différents en matière de formation initiale, d'expérience professionnelle, de culture et de niveau de français. Une activité d'introduction s'est donc avérée nécessaire afin de permettre aux étudiants d'avoir une vision d'ensemble de l'intégralité des connaissances scientifiques et méthodologiques à acquérir et de leur donner de nombreuses occasions d'apprendre à se connaître afin qu'ils puissent ensuite rapidement créer, de manière autonome, des groupes qui fonctionnent sans conflits.

Pour prendre en compte les niveaux de chacun des étudiants, ainsi que pour quitter l'aspect rébarbatif lié à la découverte de systèmes informatiques complexes, le jeu de rôle avait pour objectif de donner de la signifiance à l'activité (Viau, 1998) et d'intégrer les étudiants dans une démarche projet, pour agir sur différents leviers de motivation. D'ailleurs, la recherche en pédagogie dans ce domaine s'accorde sur le fait que la ludification est un allié important de l'enseignement de l'informatique (Guéraud et Peyrin, 1988 ; Muratet et al., 2011 ; Delozanne et al., 2011).

\section{Traduction du jeu de rôle en activité pédagogique}

Selon la Fédération Française du Jeu de Rôle [FFJdR] (2006), "Le jeu de rôle est un jeu de société coopératif. Un joueur particulier, le meneur de jeu, met en scène une aventure dans un cadre imaginaire en s'aidant d'un scénario. Les autres joueurs interprètent les personnages principaux de cette aventure. Le jeu consiste en un dialogue permanent au moyen duquel les joueurs décrivent les actions de leurs personnages. Le meneur de jeu décrit à son tour les effets de ces actions, interprète les personnages secondaires et arbitre la partie en s'appuyant sur des règles. »

Cette définition met en avant un certain nombre de propriétés qui sont intéressantes d'un point de vue pédagogique : la coopération, l'imagination, le dialogue, l'action et ses conséquences, et le respect des règles. Nous allons maintenant présenter les différents éléments à considérer 
pour mettre en place un jeu de rôle et les adaptations que nous avons choisies pour créer notre activité pédagogique.

\subsection{Le meneur de jeu}

«Le meneur de jeu, propose et arbitre les situations, interprète les rôles secondaires et garantit la cohérence du cadre fictionnel et son évolution en tenant compte en permanence des décisions des joueurs. » (FFJdR, 2006)

L'enseignant incarne le rôle du meneur de jeu. Son objectif est double : il doit construire une véritable expérience et maintenir sa cohérence tout au long de l'activité. Par véritable expérience, nous entendons une activité qui correspond à une attente ou à un questionnement de la part des étudiants. Cette expérience doit être signifiante à leurs yeux, leur proposer un défi et leur laisser l'opportunité de faire des choix en collaboration avec les autres étudiants. Il est important pour l'enseignant de maintenir la cohérence de son cadre, l'activité ne doit paraître à aucun moment factice, artificielle ou vaine (par exemple en cherchant à orienter les étudiants dans une direction quoi qu'ils fassent), cela serait contre-productif. Le rôle du meneur est de montrer les chemins possibles en utilisant les éléments présentés dans les paragraphes suivants. Par exemple, si un point théorique pose un problème à un groupe, l'enseignant n'a pas ici vocation à transmettre sa connaissance, mais à aider les étudiants à se poser les bonnes questions et à les orienter vers les ressources bibliographiques les plus pertinentes. L'objectif étant que les étudiants acquièrent l'autonomie nécessaire pour participer à la suite de l'enseignement qui se déroule sous formation d'apprentissage par projet. À noter qu'il n'est pas négatif que certains étudiants tentent de s'éloigner des chemins que vous proposez, cela leur fera vivre une expérience sûrement différente de ce que vous aviez prévu. Ce qui est important alors est de réussir, au travers du jeu de rôle, à les amener à se questionner sur les conséquences de leurs choix. Par exemple, un étudiant qui va être très préoccupé par le respect du besoin du client, au point de ne pas suffisamment réfléchir à la dimension technique pourrait devoir faire face à un client mécontent, car la solution ne fonctionne pas correctement.

Le meneur de jeu a également la responsabilité de mettre en œuvre tout ce qu'il faut pour simuler l'environnement extérieur. Il est donc responsable de la mise en œuvre du scénario que nous allons détailler. 


\subsection{Le scénario}

«Le scénario [est une] sorte de trame générique présentant des lieux, des personnages et des amorces de situations. Le terme de l'histoire étant soumis aux aléas des décisions prises par les joueurs, il n'est jamais déterminé par avance. » (FFJdR, 2006)

Lors de l'élaboration du scénario, il faut garder en tête trois objectifs : donner des repères, donner un rythme et donner de la liberté. Pour donner des repères, il faut créer un contexte, un décor, ce qui peut se faire rapidement au moyen de clichés qui vont permettre de se plonger dans l'activité sans trop d'explications. Pour donner un rythme, il faut prévoir des situations variées qui vont se présenter tout au long de l'activité. Dans notre enseignement, nous proposons aux étudiants de rejoindre un cabinet de conseil qui doit prouver à un potentiel client, en une durée très courte, sa capacité à lui proposer un ensemble de tableaux de bord pour piloter son activité commerciale. Nous avons choisi un contexte futuriste où le client fait du commerce dans une société technologiquement avancée. La situation reste à la fois réaliste, mais est suffisamment exotique pour ne pas paraître ennuyeuse. Les étudiants sont répartis en $n+1$ groupes d'étudiants. Il y aura un groupe de $\mathrm{n}$ étudiants jouant le rôle des assistants à maîtrise d'ouvrage (représentant d'un client fictif) et n groupes d'étudiants (5 ou 6 au maximum) jouant le rôle d'un cabinet de conseil. La répartition des étudiants est importante, car le meneur de jeu (qui peut incarner le PDG de l'entreprise cliente) va envoyer chacun des étudiants dans le groupe du client rencontrer un des cabinets de conseil. Sur une demi-journée, une activité comprend six phases qui jouent sur une alternance entre travail en autonomie, travail de groupe et restitution à des pairs (voir annexe 1) :

1. Étude du contexte (en autonomie) et préparation de l'entretien (en groupe).

2. Entretien avec un client (un étudiant mène l'entretien, un autre l'assiste et le restant du groupe observe et prend des notes).

3. Réalisation : phase de définition du travail et de répartition des tâches (en groupe), travail en autonomie, mise en commun (en groupe).

- Les étudiants des cabinets de conseils travaillent sur la réalisation de maquettes des tableaux de bord.

○ Les étudiants jouant le rôle des clients travaillent sur la définition de critères d'évaluation des maquettes des tableaux de bord.

4. Évaluation par les pairs : les étudiants jouant le rôle de client se mettent en binôme et évaluent deux cabinets de conseils. Tous les étudiants de ces deux cabinets participent 
aux deux évaluations: pour présenter et valoriser son travail et pour observer l'évaluation de l'autre groupe.

5. Bilan : en autonomie, les étudiants font le bilan des évaluations et partagent ensuite leurs réflexions au sein de leur groupe.

6. Conclusion et restitution : les étudiants jouant le rôle de client donnent leur classement des trois meilleures productions et argumentent leurs décisions en explicitant les critères utilisés.

Enfin, pour donner de la liberté il faut distribuer des ressources variées, définir différentes interactions possibles et donner des objectifs tout en laissant aux étudiants le choix sur la manière de les atteindre.

\subsection{Les personnages}

Les personnages sont les rôles que vont incarner les étudiants. Dans notre activité, chaque cabinet de conseil rassemble au moins un manager, un expert fonctionnel, un consultant expert en intégration de données et un consultant expert en tableaux de bord. Le groupe client ne comporte quant à lui que des assistants à maîtrise d'ouvrage. En début de séance, les rôles de manager et d'assistants à maîtrise d'ouvrage sont distribués tout d'abord aux volontaires. Ensuite, dans chaque cabinet de conseil, les étudiants choisissent le rôle qui leur convient. Le manager pouvant avoir le dernier mot en cas de problème.

Chaque étudiant récupère une fiche correspondant à son rôle qui décrit de manière précise sa mission et les ressources à sa disposition (voir Annexe 2). Parfois, les personnages ont des compétences que les étudiants n'ont pas. Pour résoudre ce problème, on peut leur donner accès à des ressources spécifiques ou leur permettre des interactions différenciées de celles des autres rôles. Enfin, il ne faut pas oublier que les enseignants ne sont pas «hors-jeu», ils doivent incarner des rôles qui s'inscrivent de manière cohérente dans l'activité.

\subsection{Les joueurs}

Les étudiants sont les joueurs d'un jeu dont ils ne connaissent pas encore les règles et cela peut leur faire perdre leurs repères au début. Il est donc nécessaire de préparer un document qu'ils devront lire en groupe et qui explique la nature de l'activité. Les joueurs joueront en équipe ; lesquelles s'opposeront à d'autres équipes. Cela va créer une zone de confiance au sein des groupes et une compétition saine entre les groupes. Il ne faut pas hésiter à proposer un prix symbolique pour le groupe vainqueur afin de stimuler la compétition. 


\subsection{Les ressources}

Les ressources doivent rassembler les connaissances à acquérir pour permettre aux étudiants d'atteindre leurs objectifs. Elles doivent être en cohérence avec les rôles incarnés par les étudiants et en quantité suffisante. Il faut veiller à ne pas trop en donner en une seule fois, car certaines seraient alors négligées.

\subsection{Les objectifs}

Le moteur d'un jeu de rôle est l'objectif à accomplir. Cet objectif est commun au groupe, mais il est possible que chaque personnage en ait également un qui lui est propre. Il est intéressant ici de mettre en œuvre cette dualité. Pour cela, il faut veiller à ce que chaque étudiant y trouve son compte et se sente responsable d'une partie de la réussite (ou de l'échec) de son groupe.

\subsection{L'écran du meneur}

Mener un jeu de rôle est une activité qui demande de gérer de nombreuses tâches à la fois, on la réalise souvent en ayant en main un aide-mémoire des informations utiles afin de ne pas perdre de temps à devoir les retrouver. Il est nécessaire d'avoir des fiches avec la définition des rôles de chacun ainsi que la liste des ressources attribuées et des objectifs à atteindre. Il faut également avoir un agenda minuté de l'activité, afin de pouvoir gérer le temps et ne pas déborder. Il est très difficile de gérer seul cette activité avec plus d'une vingtaine d'étudiants, mais à deux il est possible d'en gérer une soixantaine en interagissant avec les rôles clefs.

\section{Cadre de référence et méthodologie de l'étude}

Un des principes fondateurs du jeu de rôles est d'agir sur les leviers de la motivation de Viau (1998), et notamment celui consistant à rendre l'enseignement significatif. La première hypothèse que nous avons formulée était en effet que le jeu de rôle permettrait de donner aux étudiants une vision relativement claire des réalités professionnelles qui les attendent afin qu'ils trouvent de l'intérêt dans l'activité. Viau (1998) fait mention de l'importance pour l'étudiant de trouver un intérêt et une utilité (notamment professionnels) dans les enseignements qu'il reçoit. C'est sur ce levier que le jeu de rôle entend jouer afin de permettre aux étudiants de se projeter dans un univers professionnel simulé.

Pour vérifier les deux hypothèses formulées au début de cet article, nous avons demandé à 49 étudiants de répondre à un questionnaire comportant 5 questions sur l'activité sous forme de 
jeu de rôle (voir Annexe 3). Une question sur l'utilité perçue de cette activité (question 3), une question sur la perception des étudiants des compétences développées par l'activité (question 4), une question conditionnelle sur l'influence de l'activité sur le choix du groupe (pour le projet que les étudiants ont réalisé après l'activité) en fonction du fait que l'étudiant avait déjà un choix en tête ou non (questions 5 et 6 ) et une question sur l'efficacité perçue du travail en groupe (question 7).

Ensuite, quatre questions étaient posées pour caractériser l'étudiant en fonction de son sexe, de sa formation, de son cursus avant l'arrivée à l'école et de son expérience professionnelle.

\section{Le jeu de rôle pour mettre en scène une situation professionnelle et intérioriser un processus global}

\subsection{Le jeu de rôle comme outil de mise en situation professionnelle et levier de motivation}

À partir des données relevées dans le questionnaire (voir Annexe 3), on constate que les étudiants ont perçu un intérêt certain pour l'activité (Figure 1), avec 35 étudiants qualifiant l'activité de très à moyennement intéressante, et seulement 14 la qualifiant de peu ou très peu intéressante. En croisant certaines données, nous avons cependant remarqué un lien intéressant entre l'utilité professionnelle perçue et l'expérience professionnelle des étudiants.
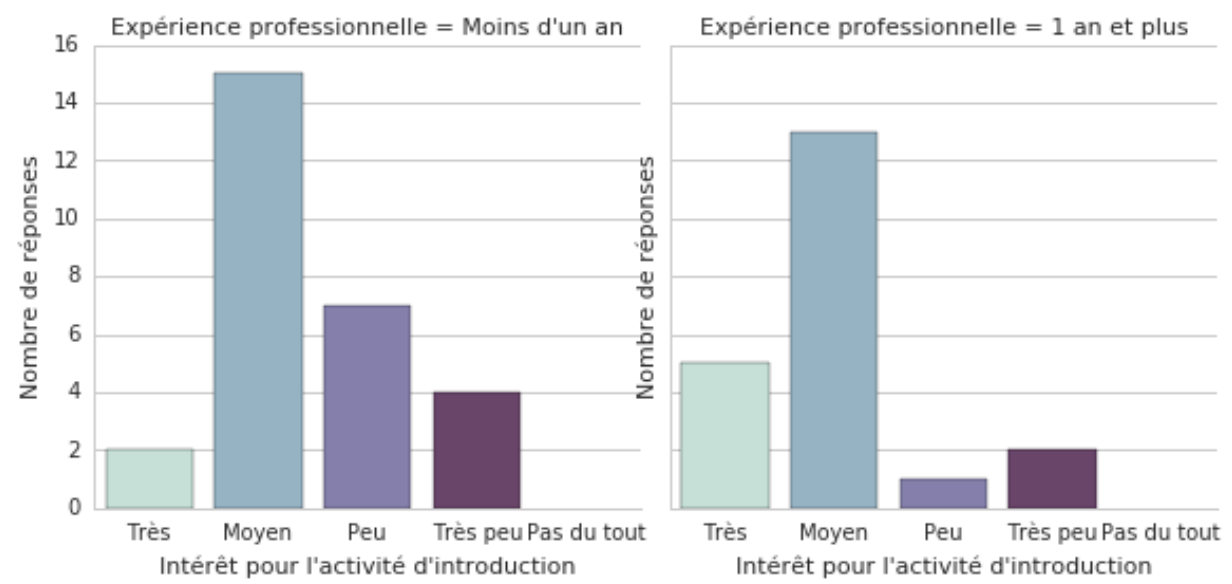

Figure 1 : Intérêt pour le jeu de rôle en fonction de l'expérience professionnelle de l'étudiant

Ainsi, on remarque que plus les étudiants ont passé de temps en situation professionnelle, plus l'activité a d'intérêt (Figure 1) et d'utilité professionnelle (Figure 2) pour eux. 

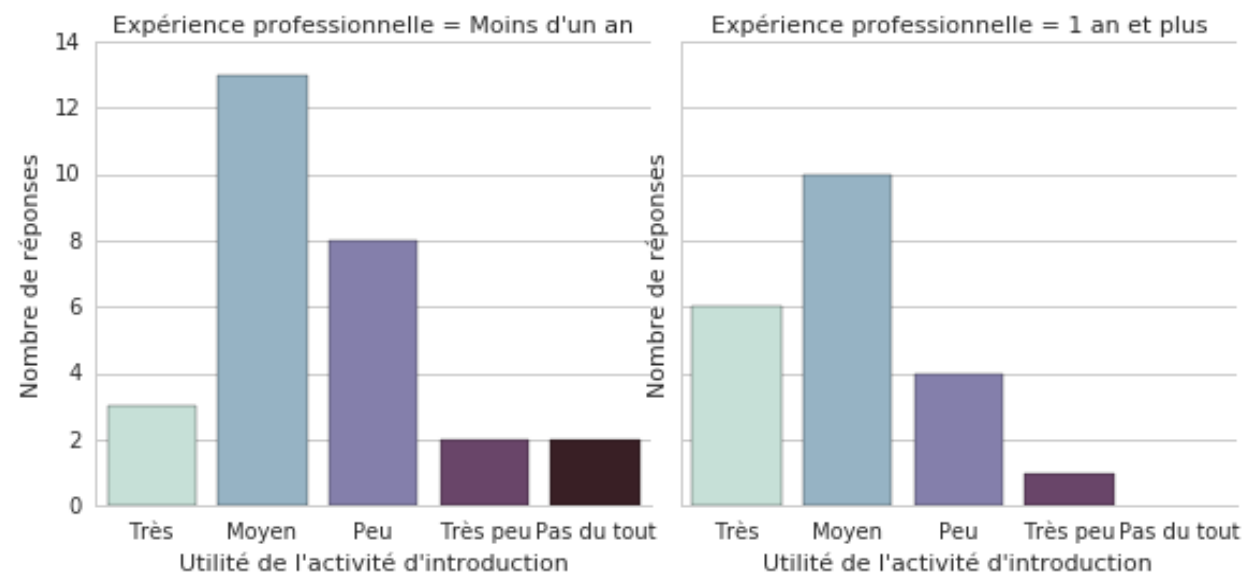

Figure 2 : Utilité professionnelle perçue du jeu de rôle

\subsection{Le jeu de rôle pour développer une vision globale de l'UV et de son contenu}

Un des objectifs de la mise en place de ce dispositif était aussi de donner une vision globale de l'enseignement aux étudiants. En effet, il semble que, comme dans beaucoup d'enseignements en informatique, les étudiants soient confrontés à la réalisation d'une tâche complexe dont ils ne comprendront la finalité qu'une fois le projet abouti. Cette situation donne lieu à de fausses routes ou à des sentiments de frustration entravant parfois la motivation pour le projet. En 2011, Muratet et al. met en exergue le fait que les étudiants en informatique doivent maîtriser des concepts et des connaissances que l'on ne peut vraiment maîtriser que lorsque l'on sait programmer, et ce dès le début de l'enseignement. Ainsi, une des solutions semble être de faire travailler directement les apprenants sur des tâches réalistes et qui ont un haut degré de signifiance (Viau, 1998) pour eux (Greitzer et al., 2007).

L'avis des étudiants est ici plus partagé avec seulement un peu plus de la moitié d'entre eux ressentant une utilité pour la réalisation de leur projet (Figure 3). Dans le questionnaire, la question était posée en expliquant que l'utilité attendue se situait au niveau des méthodes (travail en groupe, recherches bibliographiques), de l'importance de la compréhension du besoin client et de l'architecture du système décisionnel. 

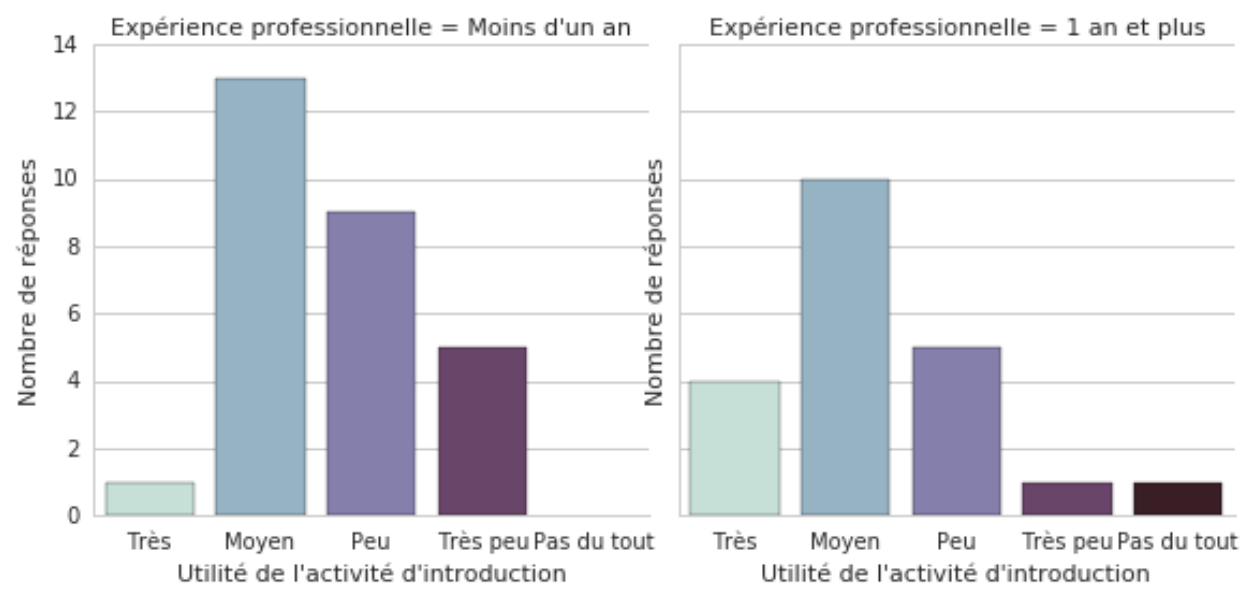

Figure 3 : Utilité perçue du jeu de rôle pour la réalisation du projet ayant lieu dans la suite de l'enseignement

Le fait qu'un peu plus de la moitié des étudiants considère le jeu de rôle très ou moyennement utile pour le déroulement de sonprojet confirme le bien-fondé de l'intention pédagogique des enseignants. Par ailleurs, le ressenti de l'équipe pédagogique est, qu'après la mise en place du jeu de rôle, les étudiants ont mieux réussi à gérer la complexité du projet qui leur était proposé. Cependant, afin d'expliquer pourquoi presque une moitié des étudiants juge le jeu de rôle peu ou pas utile pour son projet, nous pouvons avancer deux hypothèses. La première est liée à la composition de la promotion : le public étant hétérogène les étudiants ayant déjà une expérience professionnelle et certaines des compétences attendues ne trouveraient pas d'utilité à l'activité proposée. La deuxième est liée à la capacité de prise de recul de l'étudiant, il est difficile d'évaluer l'impact d'une activité sur son comportement et encore plus d'imaginer ce qui se serait passé si l'activité n'avait pas eu lieu.

Concernant la première hypothèse, la figure 3 semble permettre de la réfuter : en effet, les étudiants ayant eu une première expérience professionnelle de plus d'un an sont $66 \%$ à juger l'activité moyennement ou très utile contre $50 \%$ pour les étudiants ayant une expérience professionnelle de moins d'un an. La piste concernant la seconde hypothèse semble donc plausible : les étudiants auraient intégré les éléments nécessaires sans s'en rendre compte. Pour vérifier cela, il serait nécessaire d'explorer plus en détail les différentes utilités du jeu de rôle et de compléter la démarche par une série d'entretiens semi-directifs qui permettraient d'aider les étudiants à se questionner sur leurs pratiques avant et après cet enseignement. 


\section{Le jeu de rôle pour découvrir l'autre et créer des groupes qui fonctionnent}

\subsection{Découverte de l'autre et efficacité du travail en groupe}

Une autre ambition du dispositif était de permettre aux étudiants de travailler en groupe pour découvrir des compétences propres à des pairs qu'ils ne connaissent pas forcément bien ou pas du tout. De ce fait, le questionnaire demandait aux étudiants de choisir les trois propositions qu'ils jugeaient les plus pertinentes par rapport à leur ressenti des effets du dispositif.

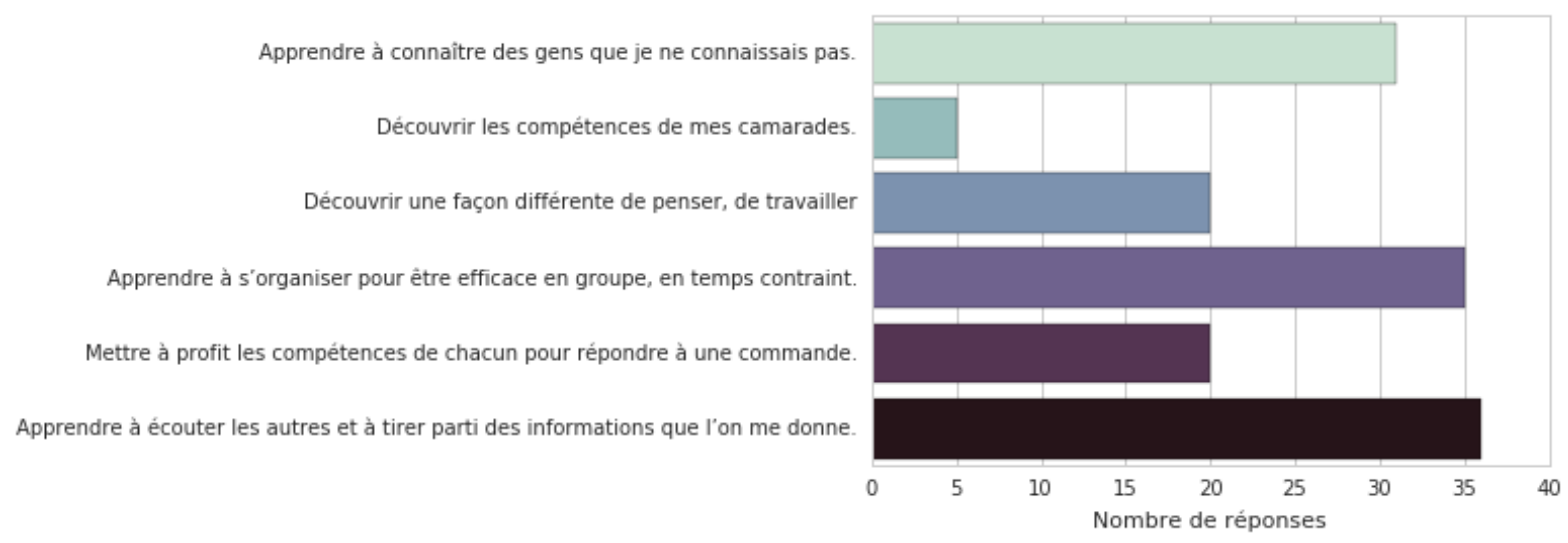

Figure 4: Propositions décrivant au mieux le jeu de rôle

Nous pouvons constater (Figure 4) que la proposition la moins choisie est «Découvrir les compétences de mes camarades » alors même que «Apprendre à connaître des gens que je ne connaissais pas » fait partie des 3 plus choisies. Par ailleurs, on constate que «Mettre à profit les compétences de chacun » est derrière des propositions plus ciblées telles que «Apprendre à s'organiser » ou «Apprendre à écouter les autres ». Il semble donc que les étudiants ne soient pas encore engagés dans une attitude réflexive sur l'acquisition des compétences nécessaires pour répondre au problème posé.

Ainsi, nous formulons l'hypothèse que les étudiants sont davantage dans une réflexion autour des relations sociales ou amicales, que dans une approche d'identification des compétences. Ils préfèrent ainsi travailler avec un groupe d'amis préexistant plutôt qu'avec une équipe présentant des compétences variées pouvant conduire à une meilleure réussite de leur projet. Valider cette hypothèse nécessiterait, ici encore, de préciser notre questionnaire ou de mener des entretiens avec les étudiants pour mieux comprendre leurs réponses à cette question. Mais ce qui intéressera l'enseignant avant tout est la raison qui conduit les étudiants à peu s'intéresser aux compétences. Notre école vivant actuellement une réforme pédagogique pour mettre en œuvre 
une véritable approche par compétences tout au long du cursus, nous pourrons étudier dans des travaux futurs l'impact de la mise en œuvre de cette démarche sur les réponses des étudiants, ce qui pourra donner un éclairage nouveau à cette problématique.

\subsection{Influence bénéfique sur la constitution des groupes}

Les derniers résultats que nous présentons ici mesurent l'influence du dispositif sur les groupes constitués à la fin de l'activité d'introduction et pour le restant de l'enseignement. Nous considérons que le jeu de rôle a eu une influence importante (réponse « oui » dans la Figure 5) sur le choix du groupe en fusionnant les réponses aux questions 5 et 6 qui étaient exclusives (pour différencier les étudiants ayant déjà fait leur choix de groupe des autres) de la manière suivante :

- Les étudiants ayant déjà fait un choix de groupe avant l'activité et déclarant que le jeu de rôle les a confortés dans leur choix ou a modifié totalement leur choix (réponse à la question 5).

- Les étudiants n'ayant pas déjà fait leur choix de groupe avant l'activité et déclarant que le jeu de rôle les a beaucoup aidés à constituer leur groupe (réponse à la question 6).

Seules les réponses «N'a eu aucune influence» (question 5) et «Pas du tout » (question 6) ont été considérées ici comme un «Non», les autres choix correspondant à une influence partielle.
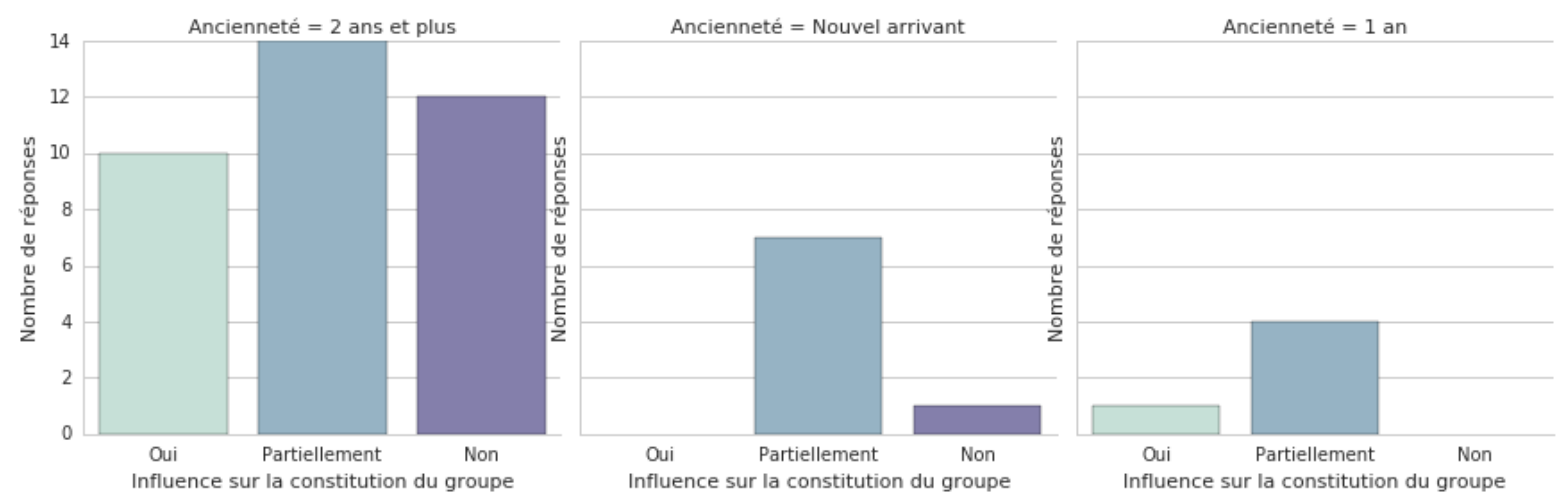

Figure 5: Influence du jeu de rôle sur la constitution du groupe en fonction de l'ancienneté de l'étudiant à l'école

Nous pouvons observer sur la Figure 5, l'impact de l'ancienneté des étudiants sur la constitution des groupes. Nous avons pu observer que, pour les étudiants présents depuis deux ans, l'activité les conduit à confirmer leur choix de continuer à travailler avec des étudiants qu'ils connaissent déjà. Par ailleurs, l'activité semble avoir pris plus d'importance pour les étudiants nouvellement arrivés puisque 12 sur 13 étudiants disent avoir ressenti l'influence du jeu de rôle sur la 
constitution des groupes. On peut ainsi conclure qu'il s'agit d'une activité particulièrement adaptée à la prise en compte de l'altérité en enseignement, dans la mesure où elle fait office de brise-glace pour les nouveaux étudiants, tandis qu'elle semble avoir un effet modéré en altérant légèrement ou en confirmant les choix de constitution de groupes pour les étudiants qui se connaissent déjà.

\section{Conclusion}

Comment concevoir un dispositif qui permettrait à la fois de prendre en compte l'altérité des niveaux et des parcours des étudiants, tout en donnant une vision d'ensemble des enjeux liés à un enseignement de spécialité en informatique ? Nous avons tenté de répondre à cette question dans le cadre d'une formation à la «Business Intelligence » en école d'ingénieur en proposant une activité sous forme d'un jeu de rôle pour introduire cet enseignement. Nous avons présenté une approche afin de transposer le jeu de rôle de loisirs en activité pédagogique en décrivant les ressources nécessaires et la posture à adopter par l'enseignant.

Nous avons fait les hypothèses suivantes : le recours au jeu de rôle permettrait de motiver les étudiants et de donner une vision d'ensemble du contenu de l'enseignement aux étudiants ; le recours au jeu de rôle aurait un impact bénéfique sur la constitution des groupes de travail en facilitant la gestion de l'hétérogénéité des étudiants. Afin de les vérifier, un questionnaire a été proposé aux 49 étudiants ayant suivi cet enseignement.

L'analyse du questionnaire nous a permis de confirmer le ressenti des enseignants sur le fait que la ludification est un moyen efficace d'aborder les notions complexes liées à la «Business Intelligence ». Notre hypothèse concernant l'impact bénéfique (en matière d'intérêt et d'utilité) est validée bien que les perceptions soient très dépendantes de l'expérience professionnelle préalable des étudiants. Nous avons partiellement validé notre hypothèse selon laquelle le jeu de rôle permet de donner une vision d'ensemble des finalités et du déroulement de l'enseignement. Pour évaluer cette hypothèse, nous nous sommes intéressés à l'utilité perçue de cette activité pour le déroulement du projet. Seule une moitié des étudiants a considéré le jeu de rôle utile à cette fin. Pour autant, le résultat reste satisfaisant, mais nécessite des investigations que nous mènerons dans les prochaines occurrences de cet enseignement pour comprendre s'il s'agit d'un manque réel d'utilité ou si, bien qu'utile, l'activité n'est pas perçue comme telle, car les étudiants manquent de recul quant à l'impact du jeu de rôle sur le 
comportement. Enfin, l'influence de l'activité sur la construction du groupe est marquée, surtout si l'étudiant a une faible ancienneté dans l'école.

Le jeu de rôle, en tant que modalité pédagogique, semble une voie intéressante à continuer d'explorer pour appréhender une matière difficile et technique comme la «Business Intelligence », mais aussi pour en découvrir l'univers professionnel.

\section{Références bibliographiques}

Brisson, L. (2015). Motivation, autonomie et évaluation : un retour d'expérience en école d'ingénieur. Colloque Questions de pédagogies dans l'enseignement supérieur (Brest, France, juin 2015), p. 391-396.

Caillois, R. (1967). Les jeux et les hommes ( $3^{\mathrm{e}}$ Éd.). Paris : Gallimard.

Delozanne, E., Jarraud, P. et Muratet, M. (2011). Un projet Jeux sérieux pour approfondir l'apprentissage de la programmation en première année à l'université. Sciences et technologies de l'information et de la communication en milieu éducatif : Analyse de pratiques et enjeux didactiques (Patras, Grèce, octobre 2011), p. 241-249.

Fédération Française du Jeu de Rôles (2006). http://www.ffjdr.org/ce-devez-savoir-jeurole/definitions-du-jeu-role/

Ginat, D., (2004). On novice Loop Boundaries and Range Conceptions. Computer Science Education, 14(3), 165-181. doi:10.1080/0899340042000302709

Greitzer, F.L., Kuchar, O.A. et Huston, K. (2007). Cognitive science implications for enhancing training effectiveness in a serious gaming context. Journal on Educational Resources in Computing, 7(3). https://doi.org/10.1145/1281320.1281322

Guéraud, V. et Peyrin, J.-P. (1988). Un jeu de rôles pour l'enseignement de la programmation. Colloque francophone sur la didactique de l'informatique (Paris, France, septembre 1988).

Muratet, M., Torguet, P., Viallet, F. et Jessel, J.-P. (2011). Évaluation d'un jeu sérieux pour l'apprentissage de la programmation. Revue d'Intelligence Artificielle, 25(2), 175-202.

Seppälä, O., Malmi, L. et Korhonen, A. (2006). Observations on student misconceptions - A case study of the Build Heap Algorithm. Computer Science Education, 16(3), 241-255.

Viau, R. (1998) La motivation en contexte scolaire ( $2^{\mathrm{e}}$ Éd.). Bruxelles : De Boeck. 


\begin{abstract}
Annexes
Vous pouvez retrouver toutes les ressources liées à la conception de cette activité de jeu et de rôle et à l'analyse des réponses au questionnaire à l'adresse suivante :

http://www.laurent-brisson.fr/project/pedagogy/
\end{abstract}

\title{
Annexe 1 - Déroulement d'une session de jeu de rôle (extrait du guide du meneur)
}

7. Préparation [40 minutes] :

○ Étude des données du système opérationnel, préparation de l'entretien avec le client et étude des bonnes pratiques en matière de tableaux de bord

○ Identification des besoins et préparation de l'entretien

8. Entretien entre un client et un cabinet de conseil (les décideurs se sont réparti les cabinets de conseil) [20 minutes]

9. Réalisation [40 minutes] :

- Cabinet de conseil : Production des spécifications fonctionnelles sous forme de maquettes de tableaux de bord

○ Client (groupe composé des $n$ décideurs) : Réflexion sur la manière d'évaluer les livrables et vos critères de choix

10. Évaluation des livrables par les clients [25 minutes] :

- Associez-vous avec un de vos collègues pour faire un binôme d'évaluateurs

- Pendant deux fois 10 minutes, vous irez écouter chacun de vos 2 groupes et devrez en ressortir avec une évaluation sur la base de la grille critériée préalablement définie.

11. Réunion-bilan [20 minutes] :

- Cabinet de conseil : Réunion-bilan au sein du groupe sur le travail effectué et les méthodes utilisées

- Client : Choix argumenté du ou des cabinets de conseil décrochant le contrat

12. Conclusion et restitution [5 minutes] 


\section{Annexe 2 - Fiche mission du rôle «Manager»}

(Indiquez ci-dessus votre Nom Prénom)

Manager

(Indiquez ci-dessus le nom de votre société)

\section{Contexte}

Dans un futur lointain, I'humanité a conquis l'espace et s'est installée dans de nombreuses galaxies. La société Jita Business, s'est épanouie en développant son activité commerciale au sein du système Jita qui se trouve au coeur de la galaxie. Jita Business achète en masse à bas coût à des producteurs qui ont un besoin rapide de liquidité, pour revendre au meilleur prix quelque temps plus tard et faire de confortables bénéfices. Dans ce contexte, les outils décisionnels sont indispensables afin de piloter l'activité marchande, analyser les activités d'achats/ventes et avoir des indicateurs sur les opérations effectuées. Jita Business vient de faire appel à vous.

\section{Notes}

(Utilisez cet espace pour noter les éléments importants)

\section{Mission Outil de pilotage}

\section{Manager Animer votre équipe pour remporter le contrat}

Vous êtes le manager d'un cabinet de conseil qui vient d'être contacté par Jita Business afin de participer à un atelier (auquel seront présents plusieurs concurrents) dans le but de proposer un prototype d'outil de pilotage. Lors de cet atelier, vous ne rencontrerez qu'un seul représentant du client afin de recueillir ses besoins. Le cabinet de conseil qui donnera le plus satisfaction sera choisi pour réaliser le projet.

Objectifs Au cours de cette activité vous devrez :

- répondre au mieux aux exigences du client pour obtenir le contrat,

- gérer votre équipe (assurez-vous d'une bonne répartition des tâches et de vos ressources humaines par rapport à la difficulté des tâches),

- gérer votre temps (si vous êtes hors délais le client ne vous choisira pas),

- animer votre équipe (vous devrez encore travailler ensemble. assurez-vous de la cohésion),

- faire le bilan de l'expérience acquise lors du projet.

\section{Directives}

Agenda Votre société doit respecter les contraintes horaires suivantes :

- Préparation [40 minutes] : étude des données du système opérationnel, préparation de l'entretien avec le client et étude des bonnes pratiques en matière de tableaux de bord

- Entretien avec le client [20 minutes]

- Production des spécifications fonctionnelles sous forme de maquettes de tableaux de bord [40 minutes]

- Évaluation de vos livrables par le client (votre groupe présente pendant 10 min et assiste à une présentation d'un autre groupe) [25 minutes]

- Debriefing au sein du groupe et annonce du cabinet de conseil décrochant le contrat [20 minutes]

\section{Critères de succès}

\section{Satisfaction de votre client}

Le premier critère de succès est la satisfaction de votre client. Si vous êtes en retard à vos rendez-vous ou fournissez un livrable ne répondant pas à ses besoins, vous n'obtiendrez pas le contrat.

\section{Animation de l'équipe}

Le second critère de succès est l'animation de votre équipe. Un bonne gestion de projet, une bonne ambiance et une réflexion sur votre travail en fin de séance assureront la réussite de vos réalisations futures. 


\section{Ressources}

Vous avez accès à de nombreuses ressources : vous devez essayer de répartir leur lecture avec vos collègues et en faire une synthèse afin de guider les choix de votre équipe. Un bon point de départ est de consulter le site de l'UV :

http://formations.telecom-bretagne.eu/bi/.

Vous pouvez également consulter de nombreux ebook acquis par la bibliothèque sur la page suivante:

http://formations.telecom-bretagne.eu/bi/bi_bibliographie.html.

Votre équipe est composée au minimum d'un expert fonctionnel, d'un expert en intégration de données, d'un expert en tableaux de bord et d'un manager.

\section{Besoin client}

Les objectifs donnés à votre expert fonctionnel sont les suivants :

- préparer le guide d'entretien et mener l'entretien pour recueillir le besoin métier exprimé par le client,

- contribuer à la conception des indicateurs en vous assurant de leur pertinence par rapport aux besoins exprimés par le client,

- vous assurer de la cohérence des tableaux de bord proposés par rapport aux besoins exprimés par le client au niveau du choix des indicateurs et du choix de leur représentation.

\section{Bases de données}

Les objectifs donnés à votre expert en intégration de données sont les suivants:

- répondre à toutes les questions de votre équipe sur la nature des données,

- valider la création d'un indicateur par rapport à la disponibilité des données dans le système opérationnel.

\section{Tableaux de bord}

Les objectifs donnés à votre expert en tableaux de bord sont les suivants :

- contribuer à la conception des indicateurs en vous assurant de la pertinence des représentations par rapport aux spécifications,

— vous assurer de la qualité des tableaux de bord proposés en terme de cohérence interne et d'accès à l'information. 
Annexe 3 - Questionnaire

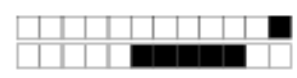

$+1 / 1 / 60+$

Questionnaire - Business Intelligence

Indiquez votre numéro d'étudiant ci-dessous, un chiffre par ligne :

Au début de cette UV, vous avez été amenés à participer à une activité d'introduction constituée de deux après-midi de jeux de rôles. Cette activité avait plusieurs objectifs pédagogiques, afin de mesurer la réussite et les améliorations à apporter à cette dernière, merci de répondre aux questions suivantes.

L'activité d'introduction avait pour but de vous mettre en situation professionnelle.

Question 1 Evaluez wotre intérét pour cette activité
Moyennement intéressante
Très peu intéressante
Pas du tout intéressante
Un peu interessante.
Très intéressante.

Question 2 A quel point jugez vous cette activité utile pour la suite de votre formation et/ou votre avenir professionnel?
Très peu utile
Pas du tout utile.
Moyennement utile
Un peu utile.
Très utile.

L'activité d'introduction avait pour but de vous présenter une vision globale :

- des méthodes et de l'approche pédagogique (travail en groupe, recherches biblio),

- de l'importance de la compréhension du besoin client dans le cadre d'un projet décisionnel,

- de l'architecture d'un système d'information décisionnel.

Question 3 Diriez-vous que l'activité d'introduction vous a été utile pour vous guider dans la mise en oeuvre de votre projet de l'UV?
Très peu utile
Un peu utile.
Très utile.
Pas du tout utile
Moyennement utile. 
Question 4 \& Cette activité vous a permis de : (sélectionnez 3 items parmi les propositions suivantes)

Mettre à profit les compétences de chacun pour répondre à une commande.

Apprendre à connaitre des gens que je ne connaissais pas.

Découvrir les compétences de mes camarades.

Découvrir une façon différente de penser, de travailler (liée à une culture, une expérience professionnelle ou une formation différente).

Apprendre à s'organiser pour être efficace en groupe, en temps contraint

Apprendre à écouter les autres et à tirer parti des informations que l' on me donne.

Aucune de ces répanses n'est correcte.

L'activité d'introduction de I'UV avait pour but de vous aider à constituer des groupes efficaces pour le reste de l'UV.

Répondez soit à la question 5 soit à la question 6 suivant la condition donnée.

Question 5 Si vous aviez, avant l'activité d'introduction, I' intention de vous mettre en groupe avec quelques-uns de vos amis. Diriez-vous que l' activité a :

Conforté wotre choix.

Modifie totalement votre choix.

Modifié partiellement votre choix.

$\mathrm{N}$ a eu aucune influence sur votre choix.

Question 6 Si vous n'aviez pas d'intention claire de wous mettre avec certaines connaissances (ou que vous ne connaissiez personne), diriez-vous que l'activité vous a aidé à constituer wotre groupe

Pas du tout.

Un peu

Très peu.

Beaucoup.

Moyennement

Question 7 Actuel lement, diriez-vous que votre groupe travaille de manière efficace?

Très efficace.

Moyennement efficace.

Très peu efficace

Un peu efficace.

Pas du tout efficance.

Dites nous en un peu plus sur vous.

Question 8 Sexe

$\bigcirc$ Fernme

Homme

Question 9 Formation actuel le

CSDS

$\bigcirc$ FIG

$\bigcirc$ ecotic

IADBA

ISPMC 
Question 10 Admission

PSI

$\bigcirc P C$

MP

Autre

AST

Question 11 Expérience professionnelle

moins d'un an

plus de 2 ans

○ 1 a 2 ans

Question 12 Accepteriez-vous d'être recontacté pour un entretien qualitatif ? Oui (et je fais attention à bien renseigner mon numéro, nom et prénom)

Non (et je peux au choix laisser ou non ce questionnaire anonyme) 